\title{
Network pharmacology exploration reveals the bioactive compounds and molecular mechanisms by which Gui-Qi-Yang-Xin Decoction suppresses myocardial fibrosis
}

Hugang Jiang ( $\sim 980828279 @ q q . c o m$ )

Gansu University of Traditional Chinese Medicine

Wenyan Lin

Gansu Provincial Hospital

Xinqiang Wang

Gansu University of Traditional Chinese Medicine

Kai Liu

Gansu University of Traditional Chinese Medicine

Zhaoyuan Fu

Gansu University of Traditional Chinese Medicine

Ming Wang

Gansu University of Traditional Chinese Medicine

Miaomiao Liu

Gansu University of Traditional Chinese Medicine

\section{Xinke Zhao}

Gansu University of Traditional Chinese Medicine

\section{Yingdong Li}

Gansu University of Traditional Chinese Medicine

\section{Research Article}

Keywords: GuiQiYangXin Decoction, myocardial fibrosis, network pharmacology, mechanism, bioactive compounds

Posted Date: January 20th, 2022

DOI: https://doi.org/10.21203/rs.3.rs-1263524/v1

License: (9) This work is licensed under a Creative Commons Attribution 4.0 International License.

Read Full License 


\section{Abstract}

Background: Gui-Qi-Yang-Xin Decoction (GQYXD), a Chinese medicine formula, has been shown to exert obvious therapeutic effects on myocardial fibrosis (MF). However, the bioactive compounds and underlying pharmacological mechanisms remain to be elucidated.

Methods:The present study aimed to determine the bioactive compounds and potential action mechanisms of GQYXD in the treatment of MF using an integrated network pharmacology approach. All compounds were identified from the corresponding database and active compounds were selected according to their oral bioavailability and drug-likeness index. The components of GQYXD and the corresponding drug targets were retrieved through drug-target databases and the potential MF genes were obtained from the GeneCard database. The abovementioned ingredients and related targets were used to construct 'compound-target-disease', protein-protein interaction (PPI) and target-pathway networks. Functional and pathway enrichment analyses were performed to determine the key biological processes and important pathways using the clusterProfiler package in R programming language. Experimental validation was performed using haematoxylin-eosin staining, Masson's trichrome staining and immunohistochemistry analysis in isoproterenol-induced MF rats, and western blot analysis, phalloidin staining and immunofluorescence staining were used to verify the proposed mechanism of protection on H9C2 cells.

Results: Fifty-five bioactive components and 59 putative targets that were directly related to GQYXD and MF were collected. The results of functional enrichment analysis revealed that responses to lipopolysaccharides, oxidative stress, hypoxia, nutrient levels and mechanical stimuli were the key biological processes and were regulated simultaneously by six direct targets, including prostaglandinendoperoxide synthase 2 (PTGS2), mitogen-activated protein kinase 14 (MAPK14), protein kinase 1 (AKT1) mitogen-activated protein kinase 8 (MAPK8), interleukin-6 (IL-6) and interleukin-1 $\beta$ (IL-1 $\beta$ ). KEGG pathway enrichment analysis found that five signalling pathways were closely related to MF treatment by GQYXD. Animal experiments showed that GQYXD could significantly alleviate isoproterenol-induced MF. In vitro cell experiment results showed that GQYXD treatment could ameliorate organisational damage to the cell cytoskeleton in $\mathrm{H} 9 \mathrm{C} 2$ induced by isoproterenol. Down-regulated expression of PTGS2, MAPK14 and MAPK8 and up-regulated expression of AKT1 was demonstrated both in animal and cell experiments.

Conclusion: GQYXD could alleviate MF based on multiple components, targets and pathways. This study also demonstrated the network pharmacology approach as an effective tool to reveal the mechanisms of traditional Chinese medicine (TCM) from a holistic perspective.

\section{Background}

Myocardial fibrosis (MF), which is a pre-lesion stage of heart failure (HF)[1, 2], is frequently diagnosed in adults aged 60-80 years in China as well as in younger adults[3, 4]. Many MF patients have various risk 
factors, such as hypertension, ischemic cardiomyopathy, abuse of isoproterenol and long-term thoracic radiation therapy [5-7]. Previous studies have shown that the predominant mechanism of MF is via various cytokines causing fibroblasts to become activated and transdifferentiate into myofibroblasts; these increase the production of collagen, which is then deposited in the extracellular matrix [8-11]. The pathogenesis of MF is similar to that of $\mathrm{HF}$, and patients with severe MF are more likely to have progressive HF $[12,13]$. Therefore, MF treatment and its related mechanism of action have attracted significant research attention in recent years.

Traditional Chinese medicine (TCM), which has been used clinically in Asia for more than 2,000 years, has unique anti-MF advantages due to its syndrome differentiation, treatment and holistic view $[14,15]$. Hence, TCM has been gradually accepted by non-Chinese and applied in the treatment of MF due to its renowned efficacy, the rich resources available, and fewer adverse reactions [16, 17]. GuiQiYangXin Decoction (GQYXD) is based on the famous traditional Chinese prescription DangGui-Buxue Decoction. This was created by Li Dongyuan, a famous physician of China, and has been widely applied for the treatment of various cardiovascular diseases such as coronary atherosclerotic heart disease, ischemic cardiomyopathy and heart failure [18-20]. GQYXD is composed of Angelica sinensis, Astragali radix, Lycii fructus, Polygonati rhizoma and Ginseng radix et rhizoma (at a ratio of 20:30:10:10:15, respectively) and functions to reinforce the qi and activate the blood. Previous studies have revealed that GQYXD can attenuate the deposition of collagen fibres between myocardiocytes and down-regulate the expression of IL-6 and IL-1 $\beta$ in myocardial tissue. Experimental data has also shown that GQYXD has a potential antiMF effect and can markedly improve a patient's condition. However, the molecular mechanism underpinning the efficacy of GQYXD remains unknown. The multi-component, multi-target and multieffect properties of Chinese herbal formulae such as GQYXD, and their synergistic and antagonistic interactions, makes it difficult to identify their effective mechanisms through conventional pharmacological approaches [21, 22]. Furthermore, the complex components and indistinct mechanism of GQYXD restrict its clinical application.

Along with the rapid development of bioinformatics has been the newly emerging network pharmacology approach. This is based on large databases and has become a useful tool to characterise the therapeutic mechanisms of TCM in detail from the molecular level to the pathway level through establishing ingredient-target, target-pathway and target-disease networks [23]. In the present study, computational tools and resources were used to investigate the pharmacological network of GQYXD to predict the active compounds, potential protein targets and pathways relating to MF. Additionally, the predictions were verified by in vivo experiments. A flowchart of the experimental procedures used in this study is shown in Figure 1.

\section{Materials And Methods}

\section{Data preparation}


Data on the components of Angelica sinensis, Astragali radix, Lycii fructus, Polygonati rhizoma and Ginseng radix et rhizoma were retrieved from the TCMSP database (http://lsp.nwu.edu.cn/tcmsp.php) and screened for oral bioavailability $(\mathrm{OB}) \geq 30 \%$ and drug-likeness $(\mathrm{DL}) \geq 0.18$. The resulting compounds were selected as active substances in the present study. The protein targets of the bioactive compounds in GQYXD were retrieved from the TCMSP (http://lsp.nwu.edu.cn/tcmsp.php), BATMAN (http://bionet.ncpsb.org/batman-tcm/) and TTD (http://bidd.nus.edu.sg/group/cjttd/) databases. MFrelated targets were obtained from Genecards, a database containing publicly available collections of genes and variants associated with human diseases, using the keyword 'Myocardial fibrosis'.

\section{Network construction and analysis}

An interaction network of bioactive GQYXD ingredients and putative targets for the treatment of MF was constructed based on their interaction data. Cytoscape (Version 3.7.2) was used to visualise the interaction network. The protein-protein interaction (PPI) data in this study were retrieved from six current PPI datasets, including the Biological General Repository for Interaction Datasets (BioGRID), Database of Interacting Proteins (DIP), Biomolecular Interaction Network Database (BIND), Molecular INTeraction Database (MID), Human Protein Reference Database (HPRD) and InAct. The BisoGenet 3.0 plugin in Cytoscape was then used to construct a PPI network, and the degree and betweenness centrality were analysed by the CytoNCA plugin in Cytoscape to determine the topological importance of nodes in the network. The clusterProfiler package in R programming language was used to annotate and visualise KEGG pathway and Gene Ontology (GO) terms associated with biological processes (BP), molecular functions (MF) and cellular components (CC).

\section{Herbs and reagents}

Angelica sinensis, Astragali radix, Lycii fructus, Polygonati rhizoma and Ginseng radix et rhizoma were purchased from the Affiliated Hospital of Gansu University of Chinese Medicine (Lan-zhou, China). The origin and quality of these herbs were identified according to the Chinese Pharmacopeia (2015 Edition). Prostaglandin-endoperoxide synthase 2 (PTGS2), mitogen-activated protein kinase 14 (MAPK14), protein kinase 1 (AKT1) and mitogen-activated protein kinase 8 (MAPK8) were purchased from Cell Signaling Technology Inc. (MA, USA).

\section{Animal experiments}

Male Wistar rats (200 \pm 20 g; licence number: SCXK-(A) 2019-014) were obtained from the Laboratory Animal Centre of Gansu University of Chinese Medicine. The Wistar rats were fed in the feeding environment (12:12 $\mathrm{h}$ light:dark cycle; temperature: $24 \pm 1^{\circ} \mathrm{C}$; humidity: $\left.40 \pm 5 \%\right)$. After acclimatization for 5 days, 40 Wistar rats were randomly divided into 4 groups of ten rats each. Rats in the negative control group received subcutaneous saline injection $\left(2 \mathrm{~mL} \cdot \mathrm{kg}^{-1} \cdot \mathrm{day}^{-1}\right)$ for 10 days. Rats in the model and GQYXD groups were induced in the experimental MF model by subcutaneous injection with isoproterenol (30 $\mathrm{mg} \cdot \mathrm{kg}^{-1} \cdot$ day $^{-1}$ ) for 10 days. Subsequently, the normal, negative control and model groups received normal saline each day, while rats in the GQYXD group received intragastric administration of GQYXD at 
$7 \mathrm{~g} \cdot \mathrm{kg}^{-1} \cdot \mathrm{day}^{-1}$ for 15 days. All of the above experimental operations were performed in accordance with the Guiding Principles for the Care and Use of Laboratory Animals of China. All animal studies were approved by the Ethical Committee of the Animal Experiment Centre of Gansu University of Chinese Medicine.

\section{Histopathological and immunohistochemical assays}

Myocardial tissue was collected, fixed in $10 \%$ formalin for $24 \mathrm{~h}$, transferred to $70 \%$ ethanol and embedded in paraffin before sectioning into $4 \mu \mathrm{m}$ thicknesses. Tissue sections mounted on slides were stained with haematoxylin and eosin (HE) for morphology and using a Masson's staining kit (cat. no. SBJ-0288) and VG staining kit (cat. no. SBJ-0297), both from Nanjing Senbeijia Biological Technology Co., Ltd., Nanjing, China, according to the manufacturer's instructions. The sections to be assessed by immunohistochemistry were stained with antibodies to PTGS2, MAPK14, AKT1 and MAPK8. The slides were incubated with 3,3'-diaminobenzidine substrate (Vector Labs, Burlingame, CA) and counterstained with Harris haematoxylin (Sigma, St Louis, MO). The histopathology and immunohistochemistry were examined under a light microscope (Motic BA210; Motic Medical Diagnostic Systems Co., Ltd., Xiamen, China).

\section{Preparation of medicated sera}

Fifty male Wistar rats weighing 250-300 g received intragastric administration of GQYXD (7 $\mathrm{g} \mathrm{kg}^{-1} \cdot$ day $\left.^{-1}\right)$, twice a day for 4 days. One hour after the final treatment, rats were intraperitoneally anaesthetised using pentobarbital sodium. Blood was collected from the abdominal aorta and centrifuged. Serum samples from all individual animals were pooled, filtered through a $0.22 \mu \mathrm{m}$ filter membrane, heat-inactivated at $56^{\circ} \mathrm{C}$ for $30 \mathrm{~min}$ and stored at $-80^{\circ} \mathrm{C}$ until use.

\section{H9C2 cell culture}

The H9C2 rat cardiomyoblast cell line was purchased from the Cell Bank of the Chinese Academy of Sciences (Shanghai, China) and cultured in Dulbecco's modified Eagle medium (DMEM; Gibco Laboratories, USA) supplemented with 10\% foetal bovine serum (FBS; Gibco Laboratories, USA) and 100 $\mathrm{U} \cdot \mathrm{mL}^{-1}$ penicillin and $100 \mathrm{mg} \cdot \mathrm{mL}^{-1}$ streptomycin in an atmosphere of $90 \%$ air and $10 \% \mathrm{CO}_{2}$ at $37^{\circ} \mathrm{C}$, as described previously. The medium was replaced every 2 days and the cells were digested with $0.05 \%$ trypsin when the cell density reached $80-90 \%$. $\mathrm{H} 9 \mathrm{C} 2$ cells were seeded in six-well plates or 96 -well plates and treated as appropriate for the following experiments.

The ISO model of $\mathrm{H} 9 \mathrm{C} 2$ cells was built to mimic myocardial injury in vivo. Briefly, when the $\mathrm{H} 9 \mathrm{C} 2$ cells reached $80 \%$ confluence, the culture medium was removed and $\mathrm{H} 9 \mathrm{C} 2$ cells were washed three times with $1 \%$ FBS DMEM. The cells were exposed to isoproterenol $(80 \mu \mathrm{M})$ for $48 \mathrm{~h}$ using $1 \%$ FBS DMEM to establish the cell model. After cell injury, the GQYXD group received $5 \mathrm{~mL}$ of GQYXD-medicated sera for 3 $\mathrm{h}$ and the control and model groups received $5 \mathrm{~mL}$ of FBS. 


\section{Western blot analysis.}

After the different treatments, the cells were collected and lysed in ice-cold radioimmunoprecipitation assay (RIPA; Beyotime, China) buffer containing protease inhibitors phenylmethylsulfonyl fluoride (PMSF; Beyotime, China) and protease inhibitor cocktail (Beyotime, China) and then centrifuged at 12,000 rpm at $4^{\circ} \mathrm{C}$ for $15 \mathrm{~min}$ to obtain the supernatant. Equal amounts of protein lysates were loaded onto a $5 \%-10$ $15 \%$ SDS-PAGE gel and transferred to polyvinylidene difluoride (PVDF) membrane. The membranes were blocked in $5 \%$ non-fat milk for $1 \mathrm{~h}$ at room temperature and then incubated overnight with primary antibodies against MAPK14 (1:1000), MAPK8 (1:600) and GAPDH $(1: 1,000)$. The membranes were subsequently incubated with fluorescent secondary antibody $(1: 15,000)$ for $4 \mathrm{~h}$ at room temperature. The membranes were then washed three times with TBST for 5 minutes each time. The protein bands were detected with the Gel Doc XR system (BIO-RAD, USA).

\section{Phalloidin staining}

Cells were cultured for $24 \mathrm{~h}$, fixed in $4 \%$ formalin for $20 \mathrm{~min}$, washed three times with phosphate-buffered saline (PBS), and stained with $5 \mu \mathrm{g} \cdot \mathrm{mL}^{-1}$ of phalloidin conjugate solution (P5282, Sigma, Carlsbad, CA, USA) in PBS for $40 \mathrm{~min}$ at $37^{\circ} \mathrm{C}$. The cells were washed three times with PBS to remove the unbound phalloidin conjugate and imaged by confocal laser scanning microscopy (Fluoview1000; Olympus, Tokyo, Japan).

\section{Immunofluorescence staining}

After different stimulation, $\mathrm{H} 9 \mathrm{C} 2$ cells were fixed with $4 \%$ paraformaldehyde for 30 min, washed three times with PBS and then treated with $0.2 \%$ Triton X-100 (Solarbio, China) at room temperature for 20 min. After washing three times with PBS, cells were blocked in 5\% BSA (Servicebio, China) for $30 \mathrm{~min}$ at room temperature. Subsequently, cells were incubated with primary antibodies against PTGS2 (1:100) and AKT1 (1:100) overnight at $4^{\circ} \mathrm{C}$. Samples were then incubated with anti-mouse secondary antibody (1:300) at $37^{\circ} \mathrm{C}$ for $1 \mathrm{~h}$ and then cells were stained with DAPI (Wuhan Antgene, China) for $5 \mathrm{~min}$. Images were obtained by confocal laser scanning microscopy.

\section{Results}

\section{Screening out active components of GQYXD}

To identify the active ingredients of GQYXD, components of each herb in GQYXD were collected from TCMSP databases. As shown in Supplement 1, 55 unique bioactive components of GQYXD were screened out from the 628 chemical components. Specifically, there were two bioactive components in Angelica sinensis, 13 bioactive components in Astragali radix, 23 bioactive components in Lycii fructus, eight bioactive components in Polygonati rhizoma, 15 bioactive components in Ginseng radix et rhizoma. Among these, $\beta$-sitosterol was a common component of Angelica sinensis, Lycii fructus, Polygonati 
rhizoma and Ginseng radix et rhizoma, Stigmasterol was shared by Angelica sinensis, Lycii fructus and Ginseng radix et rhizoma, and kaempferol was in Astragali radix and Ginseng radix et rhizoma.

\section{Putative targets of GQYXD for the treatment of MF}

Molecular similarity matching and database searching were used to obtain the targets of the bioactive ingredients in GQYXD. A total of 224 potential protein targets were found after eliminating the overlaps. These targets of active compounds in GQYXD were mapped with 399 candidate targets relating to MF from the GeneCard database with 'score' $\geq 10.0$, as shown in Supplement 3. This identified 59 targets of 55 components in GQYXD that were associated with MF, as shown in Figure 2, Supplement 4. These targets were considered to be the putative targets of GQYXD for the treatment of MF and used to construct a component-target network comprising 114 nodes (55 active components and 59 putative targets) and 250 edges, as shown in Figure 3.

\section{Identification of candidate targets for GQYXD against MF}

As indicated by recent evidence in network biology, disease-related genes and proteins do not work alone. Instead, there are diverse interactive pathways and molecular networks on various levels. To clarify the pharmacological mechanism by which GQTXD ameliorates MF, a protein-protein network (PPI) was constructed with 1,387 nodes and 26,914 edges, which may reflect the behaviour and properties of the biological molecules (Fig. 4A, Supplement 5). Subsequently, a topological analysis of the PPI was conducted. Nodes with topological features exceeding the median of all nodes were considered as hubs in the network, and therefore candidate targets in the present study. These candidate targets were identified according to a widely used plugin CytoNCA, based on targets with higher values of the two topological features 'Degree' and 'Betweenness centrality'. Following the construction of the PPI network, calculation of these two topological parameters for all targets identified that targets with 'Degree' $>61$ and 'Betweenness centrality' > 600 were the candidate targets of GQYXD for the treatment of MF. Ultimately, 15 direct targets were identified, as shown in Figure 4B-C.

\section{GO and KEGG pathway enrichment analysis}

To identify the biological characteristics of the 59 predicted targets of GQYXD for MF, the GO and pathway enrichment analyses were conducted via colorspace, stringi, ggplot2, DOSE, clusterProfiler and enrichplot in the R programming language package; these enable the comparison of biological themes among gene clusters that support humans via the implementation of methods to statistically analyse and visualise functional profiles (GO and KEGG) of gene and gene clusters. There were 478 biological process (BP), 20 cellular component (CC) and 22 molecular function (MF) terms in total, which met the requirements of count $\geq 2$. The detailed GO information is shown in Supplement 8-10. The top 20 significantly enriched terms in the BP, $C C$ and MF categories are shown in Figure $5 \mathrm{~A}-\mathrm{C}$, which indicated that GQYXD may exert its therapeutic effects on MF via responses to lipopolysaccharides and hypoxia, cytokine receptor binding, and tetrapyrrole binding in the cytosol, membrane raft and membrane regions. 
To explore the signal pathways of GQYXD that were potentially associated with MF, KEGG pathway analysis of involved targets was conducted. The detailed results are shown in Supplement 11 and indicated that the alleviation of MF by GQYXD was closely related to 20 pathways, including several significant signalling pathways including the TNF signalling pathway, IL-17 signalling pathway, MAPK signalling pathway and C-type lectin receptor signalling pathway, as shown in Figure 6.

\section{Isoproterenol induces myocardial fibrosis}

Isoproterenol-induced myocardial injury lesions are characterized by necrotic myocytes, inflammation and subsequent reparative fibrosis. Myocyte necrosis and obvious infiltration of inflammatory cellsmainly macrophages-into the myocardial tissue were seen in the model group. In the GQYXD group, the myocardial injury was almost recovered, but some focal scars in the affected areas were observed (Fig. 7I). Slight collagen deposition was seen in the myocardium of the normal and negative control groups. Additionally, significant collagen fibre deposition around the arterioles and metarterioles was present in the model group and thick collagen fibres were present between myocardiocytes. In most cases, the main feature was collagen interstitial fibrosis, giving a 'brindled' look to the myocardium through the alternation of areas with fibrosis and areas of myocardial cells. The extent of myocardial fibrosis in the GQYXD group was significantly smaller than in the model group, as shown in Figure 7-II, suggesting that GQYXD could alleviate MF.

\section{Isoproterenol regulates the expression of PTGS2, MAPK14, AKT1 and MAPK8}

The KEGG pathway enrichment results indicated that the TNF, IL-17, C-type lectin receptor, Toll-like receptor and VEGF signalling pathways were intimately associated with MF treatment by GQYXD. The primary targets, including PTGS2, MAPK14, AKT1, MAPK8, IL-6, IL-1 $\beta$ and GAPDH, were involved in the enrichment of these five pathways. The effect of GQYXD on AKT1, MAPK8, MAPK14 and PTGS2 protein expression was explored by immunohistochemistry analysis. As shown in Figure 7-III-VI, the results showed a significant increase in AKT1 and the down-regulated expression of MAPK8, MAPK14 and PTGS2.

\section{MAPK8 and MAPK14 are potential myocardial fibrosis targets}

To confirm that the expression of MAPK8 and MAPK14 was affected in isoproterenol-induced H9C2 cells, western blot assay was used to determine the effect on protein levels. A significant reduction in the expression of MAPK8 and MAPK14 was observed in GQYXD-treated cells compared to model group cells. There was no significant difference in the expression of MAPK8 and MAPK14 at the protein level in GQYXD-treated cells compared to control group cells (Fig. 8).

\section{Isoproterenol induces extensive changes in cytoskeleton structure}

The F-actin organisation in $\mathrm{H} 9 \mathrm{C} 2$ cells was investigated by fluorescent phalloidin staining. Control cardiomyocytes showed regular and well-defined actin organisation, while cardiomyocytes in the model group showed more diffuse and irregular F-actin disposition. The differences could be visualised in the 
representative cardiomyocytes. However, treatment by GQYXD improved F-actin organisation in cardiomyocytes compared with the model group and produced a marked remission in isoproterenolinduced cardiac hypertrophy and cardiac fibrosis, as shown in Figure 9.

\section{GQYXD ameliorates isoproterenol-aggravated injury by regulating the protein expression of AKT1 and PTGS2}

The effect of GQYXD on the expression of AKT1 and PTGS2 in isoproterenol-induced H9C2 cells was investigated. AKT1 expression was lower in the model group compared with the control group, and GQYXD reversed this effect. The expression of PTGS2 was higher in the model group compared with the control group, but the expression of PTGS2 was lower in the GQYXD group than the model group, as shown in Figure $10 \mathrm{~A}$ and $\mathrm{B}$.

\section{Discussion}

MF is closely associated with increased myocardial stiffness, cardiomyocyte necrosis, arrhythmia, sudden cardiac death and unfavourable prognosis, and plays a role in the remodelling process that leads to HF $[2,24-26]$. Previous research has shown that MF is caused by the deposition of fibrillar connective tissue between cardiomyocytes and cardiac fibroblasts [27-29]. Current Western medicine does not provide adequate therapeutic regimens for the treatment of MF. Some experimental research has revealed that transforming growth factor- $\beta$ (TGF- $\beta$ ) and galectin-3 play key roles in the development of MF and that angiotensin II and aldosterone are essential to promoting the deposition of collagen in the myocardium as a response to hypoxia, oxidative stress and lipopolysaccharides [30-34]. Thus, further exploration of the potential mechanism of MF is essential in the development of more specific, effective and less toxic MF therapies.

Previous studies have shown that GQYXD can markedly suppress the deposition of collagen in the myocardium and reduce ventricular premature beats in HF. The overall efficacy of GQYXD combined with sacubitril/valsartan in the treatment of chronic HF is as high as $70 \%$. The animal experiments in this study also showed that GQYXD could improve the pathological status of isoproterenol-induced MF rats.

In the present study, network construction approaches were used to identify the possible bioactive compounds and potential targets, and to explore the underlying mechanisms of GQYXD in the treatment of MF $[35,36]$. The results of network pharmacological analysis showed that GQYXD ameliorated MF by multi-target, multi-component regulation, reflecting the clinical advantages of GQYXD in the treatment of MF. Gene ontology (GO) enrichment analysis was used to measure gene function from biological process (BP), molecular function (MF) and cell composition (CC) perspectives. BP function was used to investigate the biological functions in which the direct targets of GQYXD were involved in the treatment of MF. The results of GO enrichment analysis showed that the direct targets of GQYXD alleviated MF mainly by participating in biological processes in response to lipopolysaccharides, oxidative stress, hypoxia, nutrient levels and mechanical stimulus. This indicated that the bioactive ingredients of GQYXD could 
directly suppress oxidative stress, improve myocardial tolerance to hypoxia and promote myocardial energy metabolism by supplementing qi and activating blood circulation.

The putative targets of GQYXD were MF, PTGS2, MAPK14, AKT1, MAPK8, as predicted by their p-values. These targets were related to the TNF, IL-17, C-type lectin receptor, Toll-like receptor and VEGF signalling pathways. Accordingly4, the biological mechanism of GQYXD in MF treatment was explored from the different perspectives of KEGG and GO enrichment analysis. However, the results predicted by network pharmacological analysis required experimental validation. The direct targets IL- 6 and IL-1 $\beta$ were verified in our previous study on the treatment of MF by GQYXD. Therefore, in the present study, the roles of PTGS2, MAPK14, AKT1 and MAPK8 in the treatment of MF were explored. In the present study, an MF rat model revealed that GQYXD may inhibit MF progression mainly via significantly down-regulation of the expression of PTGS2, MAPK14 and MAPK8, and marked up-regulation of the expression of AKT1. Meanwhile, GQYXD treatment significantly decreased the deposition of collagen in myocardial tissue. These results were in accordance with predictions based on the network pharmacology results; that is, that GQYXD exerted its anti-MF efficacy via regulating the expression of PTGS2, MAPK14, AKT1 and MAPK8.

All the above findings demonstrate a successful application of the network pharmacology approach in identifying the mechanism of action of TCM. The detailed pharmacological mechanisms by which GQYXD ameliorates MF will be investigated in a future study. In summary, this study systematically explored the pharmacological mechanisms of GQYXD in the treatment of MF via network pharmacology and animal experiments. It is also noteworthy that network pharmacology has great advantages in explaining the therapeutic mechanisms of TCM, but there is also the need for further animal experimental research.

\section{Conclusion}

The network pharmacology method was used to identify 55 active components and 59 potential targets of GQYXD in the alleviation of MF. Combined with experimental verification both in vitro and in vivo, it was found that GQYXD may attenuate MF partially by regulating targets PTGS2, MAPK14, AKT1 and MAPK8, and subsequently decreasing the deposition of collagen in myocardial tissue.

\section{Declarations}

\section{Conflict of interest}

The authors declare that the research was conducted in the absence of any commercial or financial relationships that could be construed as a potential conflict of interest.

\section{Author contributions}


$Y L$ and $X Z$ contributed towards conceiving the research. $H J$ and $X W$ conducted the animal experiments. $H W, Z Y$ and $Y W$ assisted in conducting the experiments. WL, ML, HJ and $X W$ analysed the data and drafted the manuscript. YL and $X Z$ critically revised the manuscript.

\section{Funding}

This work was supported by the National Natural Science Foundation of China (81860786), the Gansu Provincial Department of Education Science and Technology Achievement Transformation Project (2016D-11), the Capacity Building Project of Chinese Medicine (2019XZZX-XXG002), Open Subject of Shandong Province Traditional Chinese Medicine Classic Famous Collaborative Innovation Center (2019KFZ02).

\section{References}

1. Bacmeister $L$ et al (2019) Inflammation and fibrosis in murine models of heart failure. Basic Res Cardiol 114(3):19

2. González A et al (2018) Myocardial Interstitial Fibrosis in Heart Failure: Biological and Translational Perspectives. J Am Coll Cardiol 71(15):1696-1706

3. Wang H, Yang JF (2019) [Prevention and management of heart failure in China:past,present and future perspective]. Zhonghua xin xue guan bing za zhi 47(9):714-717

4. Zhang Y et al (2017) Contemporary Epidemiology, Management, and Outcomes of Patients Hospitalized for Heart Failure in China: Results From the China Heart Failure (China-HF) Registry. J Card Fail 23(12):868-875

5. Korodi S et al (2019) Impact of inflammation-mediated myocardial fibrosis on the risk of recurrence after successful ablation of atrial fibrillation - the FIBRO-RISK study: Protocol for a non-randomized clinical trial. Med (Baltim) 98(9):e14504

6. Cha MJ et al (2018) Association of cardiovascular risk factors on myocardial perfusion and fibrosis in asymptomatic individuals: cardiac magnetic resonance study. Acta Radiol 59(11):1300-1308

7. Zannad F, Rossignol P (2018) Cardiorenal Syndrome Revisited Circulation 138(9):929-944

8. Prabhu SD, Frangogiannis NG (2016) The Biological Basis for Cardiac Repair After Myocardial Infarction: From Inflammation to Fibrosis. Circ Res 119(1):91-112

9. Rodriguez $\mathrm{P}$ et al (2019) Deletion of delta-like 1 homologue accelerates fibroblast-myofibroblast differentiation and induces myocardial fibrosis. Eur Heart J 40(12):967-978

10. Lu D et al (2013) Increase in cellular cyclic AMP concentrations reverses the profibrogenic phenotype of cardiac myofibroblasts: a novel therapeutic approach for cardiac fibrosis. Mol Pharmacol 84(6):787-793

11. Rosin NL et al (2013) Regulation and role of connective tissue growth factor in Angll-induced myocardial fibrosis. Am J Pathol 182(3):714-726 
12. Wolfson AM et al (2018) Chronic heart failure management and remote haemodynamic monitoring. Heart 104(23):1910-1919

13. Park SJ et al (2019) Assessment of Myocardial Fibrosis Using Multimodality Imaging in Severe Aortic Stenosis: Comparison With Histologic Fibrosis. JACC Cardiovasc Imaging 12(1):109-119

14. Bu D et al (2019) Study of the mechanism underlying therapeutic effect of Compound Longmaining on myocardial infarction using a network pharmacology-based approach. Biomed Pharmacother 118:109234

15. Zheng $\mathrm{J}$ et al (2019) Fatal acute arsenic poisoning by external use of realgar: Case report and 30 years literature retrospective study in China. Forensic Sci Int 300:e24-e30

16. Lu W et al (2019) Qishen Granule Improved Cardiac Remodeling via Balancing M1 and M2 Macrophages. Front Pharmacol 10:1399

17. Shang $L$ et al (2019) Plantamajoside attenuates isoproterenol-induced cardiac hypertrophy associated with the HDAC2 and AKT/ GSK-3 $\beta$ signaling pathway. Chem Biol Interact 307:21-28

18. Li C et al (2019) Dangguibuxue decoction abolishes abnormal accumulation of erythroid progenitor cells induced by melanoma. J Ethnopharmacol 242:112035

19. Hu G et al (2018) Danggui Buxue decoction promotes angiogenesis by up-regulation of VEGFR(1/2) expressions and down-regulation of sVEGFR(1/2) expression in myocardial infarction rat. J Chin Med Assoc 81(1):37-46

20. Miao X et al (2018) Metabolomics analysis of serum reveals the effect of Danggui Buxue Tang on fatigued mice induced by exhausting physical exercise. J Pharm Biomed Anal 151:301-309

21. Wei S et al (2019) Network pharmacology exploration reveals the bioactive compounds and molecular mechanisms of Li-Ru-Kang against hyperplasia of mammary gland. Mol Genet Genomics 294(5):1159-1171

22. Zhang Q et al (2019) Identification of the Active Constituents and Significant Pathways of GuizhiShaoyao-Zhimu Decoction for the Treatment of Diabetes Mellitus Based on Molecular Docking and Network Pharmacology. Comb Chem High Throughput Screen 22(9):584-598

23. Zhou JL et al (2019) Chemical markers' knockout coupled with UHPLC-HRMS-based metabolomics reveals anti-cancer integration effects of the curcuminoids of turmeric (Curcuma longa L.) on lung cancer cell line. J Pharm Biomed Anal 175:112738

24. González A et al (2019) The complex dynamics of myocardial interstitial fibrosis in heart failure. Focus on collagen cross-linking. Biochim Biophys Acta Mol Cell Res 1866(9):1421-1432

25. Gyöngyösi M et al (2017) Myocardial fibrosis: biomedical research from bench to bedside. Eur J Heart Fail 19(2):177-191

26. Kong P, Christia P, Frangogiannis NG (2014) The pathogenesis of cardiac fibrosis. Cell Mol Life Sci 71(4):549-574

27. Wan YJ et al (2019) Protocatechualdehyde reduces myocardial fibrosis by directly targeting conformational dynamics of collagen. Eur J Pharmacol 855:183-191 
28. Wang L et al (2019) Chikusetsu saponin IVa attenuates isoprenaline-induced myocardial fibrosis in mice through activation autophagy mediated by AMPK/mTOR/ULK1 signaling. Phytomedicine 58:152764

29. Podlesnikar T, Delgado V, Bax JJ (2018) Cardiovascular magnetic resonance imaging to assess myocardial fibrosis in valvular heart disease. Int J Cardiovasc Imaging 34(1):97-112

30. Guo $Y$ et al (2017) Entanglement of GSK-3 $\beta$, $\beta$-catenin and TGF- $\beta 1$ signaling network to regulate myocardial fibrosis. J Mol Cell Cardiol 110:109-120

31. Anyfanti $P$ et al (2019) Association of galectin-3 with markers of myocardial function, atherosclerosis, and vascular fibrosis in patients with rheumatoid arthritis. Clin Cardiol 42(1):62-68

32. Li S et al (2019) Perindopril and a Galectin-3 Inhibitor Improve Ischemic Heart Failure in Rabbits by Reducing Gal-3 Expression and Myocardial Fibrosis. Front Physiol 10:267

33. Ding J et al (2019) Klotho inhibits angiotensin Il-induced cardiac hypertrophy, fibrosis, and dysfunction in mice through suppression of transforming growth factor- $\beta 1$ signaling pathway. Eur $\mathrm{J}$ Pharmacol 859:172549

34. Wu P et al (2019) Lovastatin attenuates angiotensin II induced cardiovascular fibrosis through the suppression of YAP/TAZ signaling. Biochem Biophys Res Commun 512(4):736-741

35. Lee AY et al (2018) Network pharmacology-based prediction of active compounds and molecular targets in Yijin-Tang acting on hyperlipidaemia and atherosclerosis. J Ethnopharmacol 221:151-159

36. Huang $\mathrm{J}$ et al (2017) Identification of the active compounds and significant pathways of yinchenhao decoction based on network pharmacology. Mol Med Rep 16(4):4583-4592

\section{Supplemental Data}

Supplements 1-11 are not available with this version.

\section{Figures}




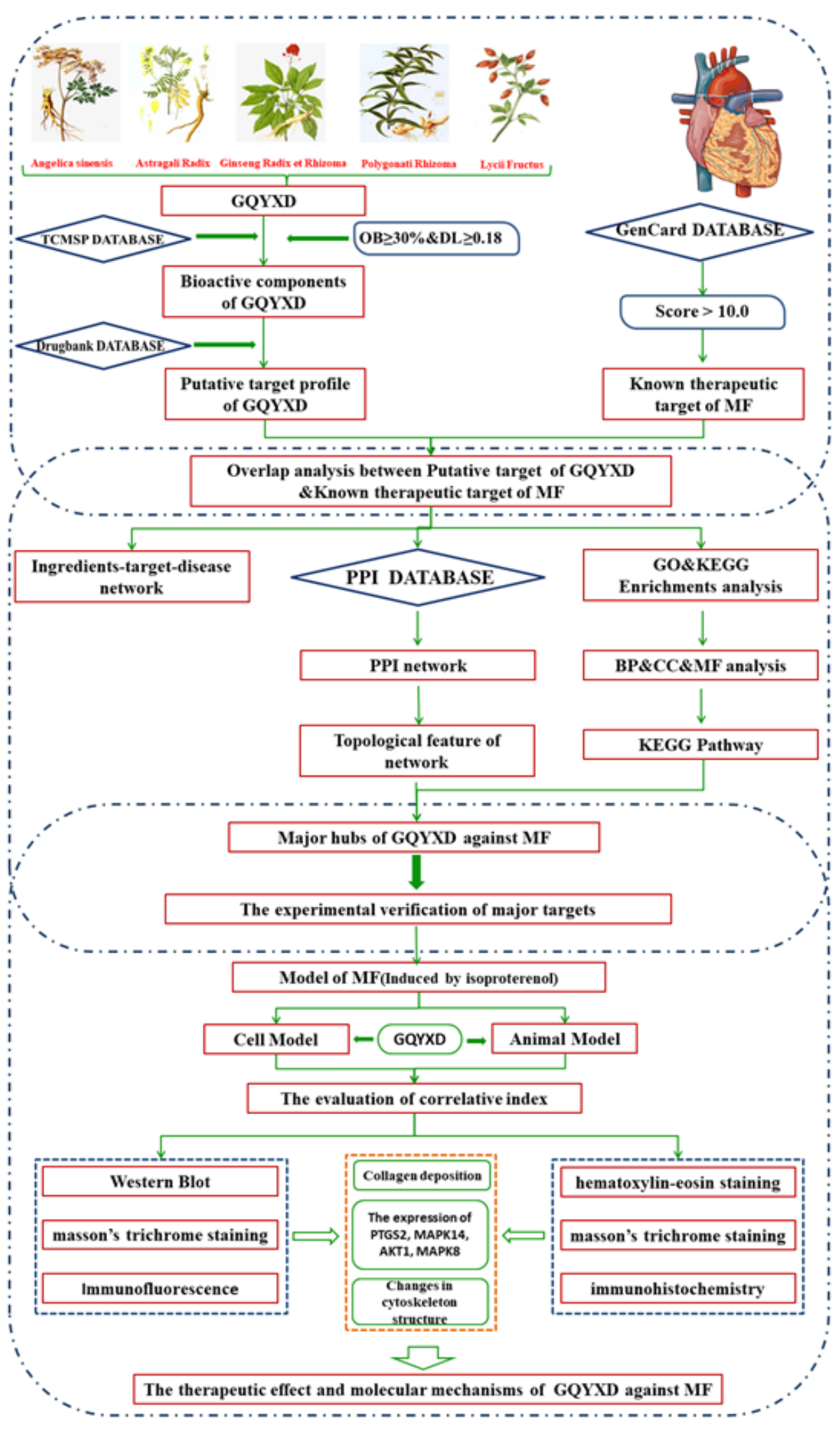

\section{Figure 1}

Flowchart of network pharmacology-based strategy for revealing the mechanisms of GQYXD against myocardial fibrosis. Abbreviations: GQYXD, Gui-Qi-Yang-Xin Decoction; TCMSP, Traditional Chinese medicine systems pharmacology; PPI, protein-protein interaction; OB: oral bioavailability; DL: druglikeness. 


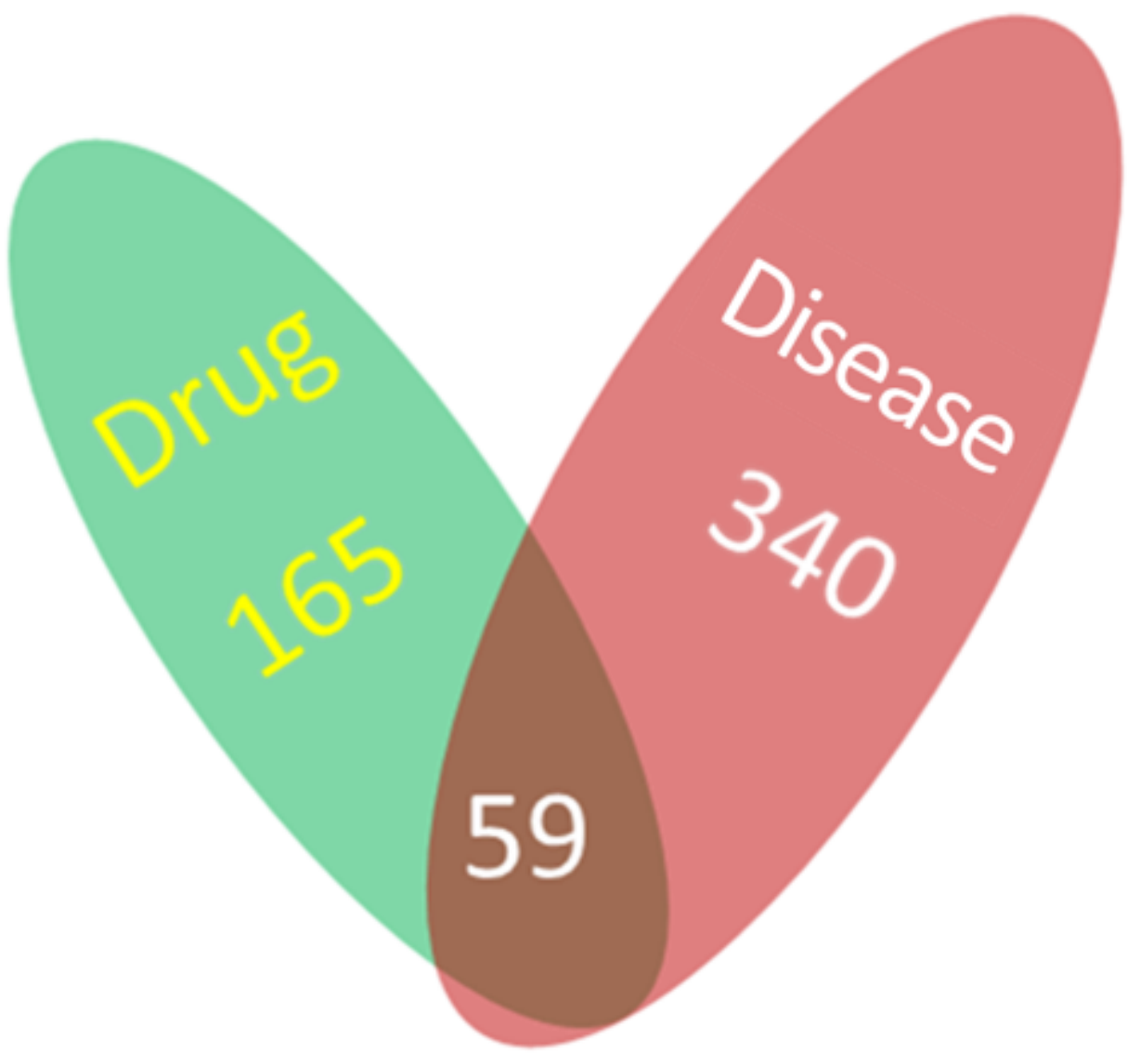

Figure 2

Venn diagram of candidate targets in GQYXD and MF 


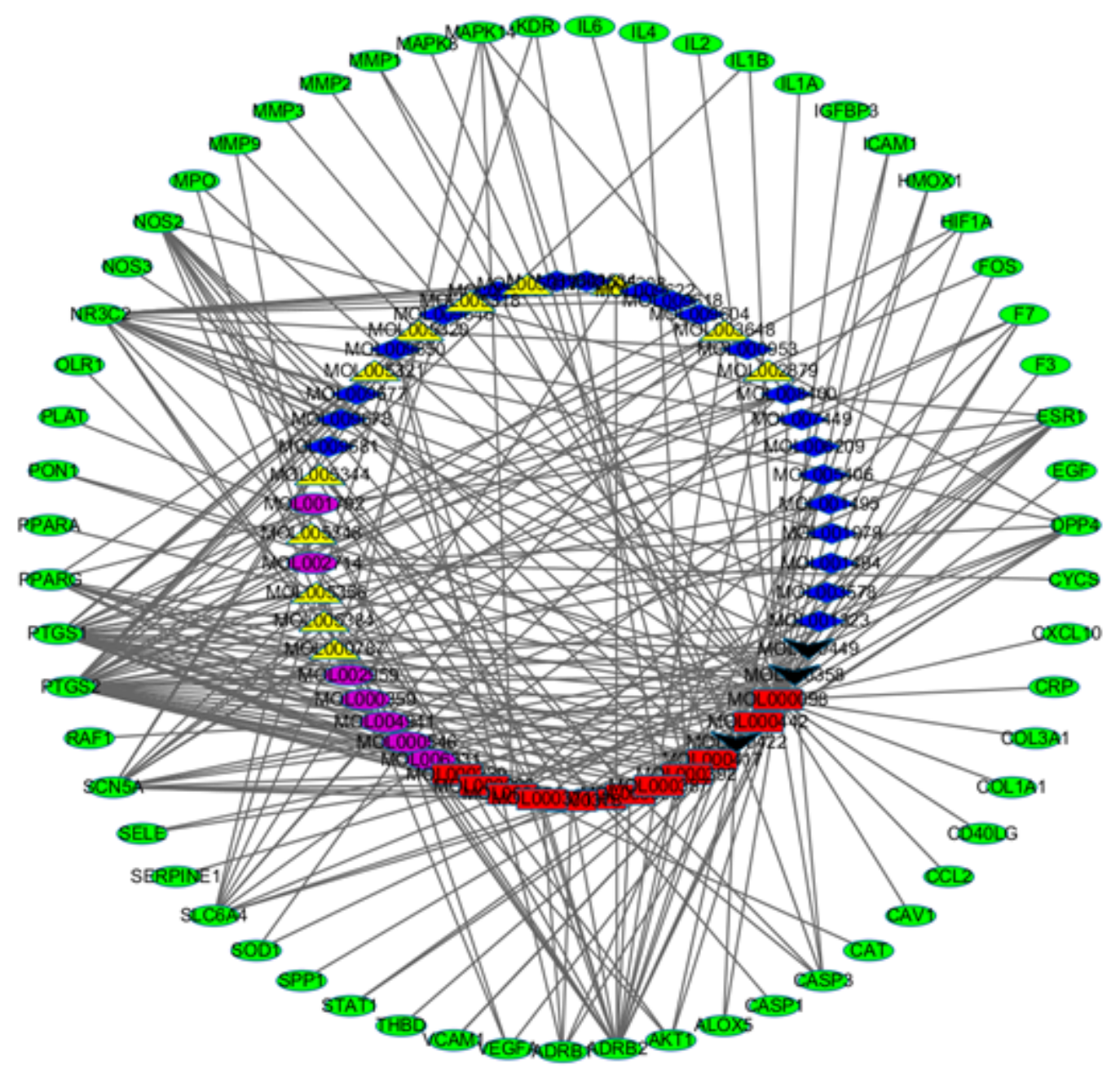

Figure 3

The component-target network. The outside circles refer to 59 putative targets of GQYXD for the treatment of MF. The inside circles represent 55 active components in GQYXD.

\section{Figure 4}

A) The PPI network was constructed by the overlap of putative GQYXD targets and known MF-related targets.

B) topological analysis of the PPI by the topological parameter of 'Degree'.

C) topological analysis of the PPI by calculating the topological parameter of 'Betweenness centrality'. 


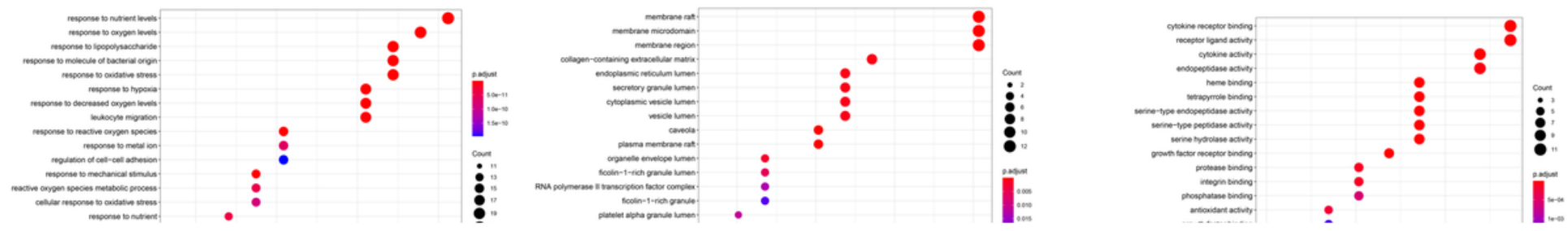

\section{Figure 5}

A) Representative bubble plots of biological process analysis of the core targets.B) representative bubble plots of cellular components of identified targets; $\mathrm{C}$ ) representative bubble plots of molecular function among candidate targets. Gene ratio = count $/$ set size.

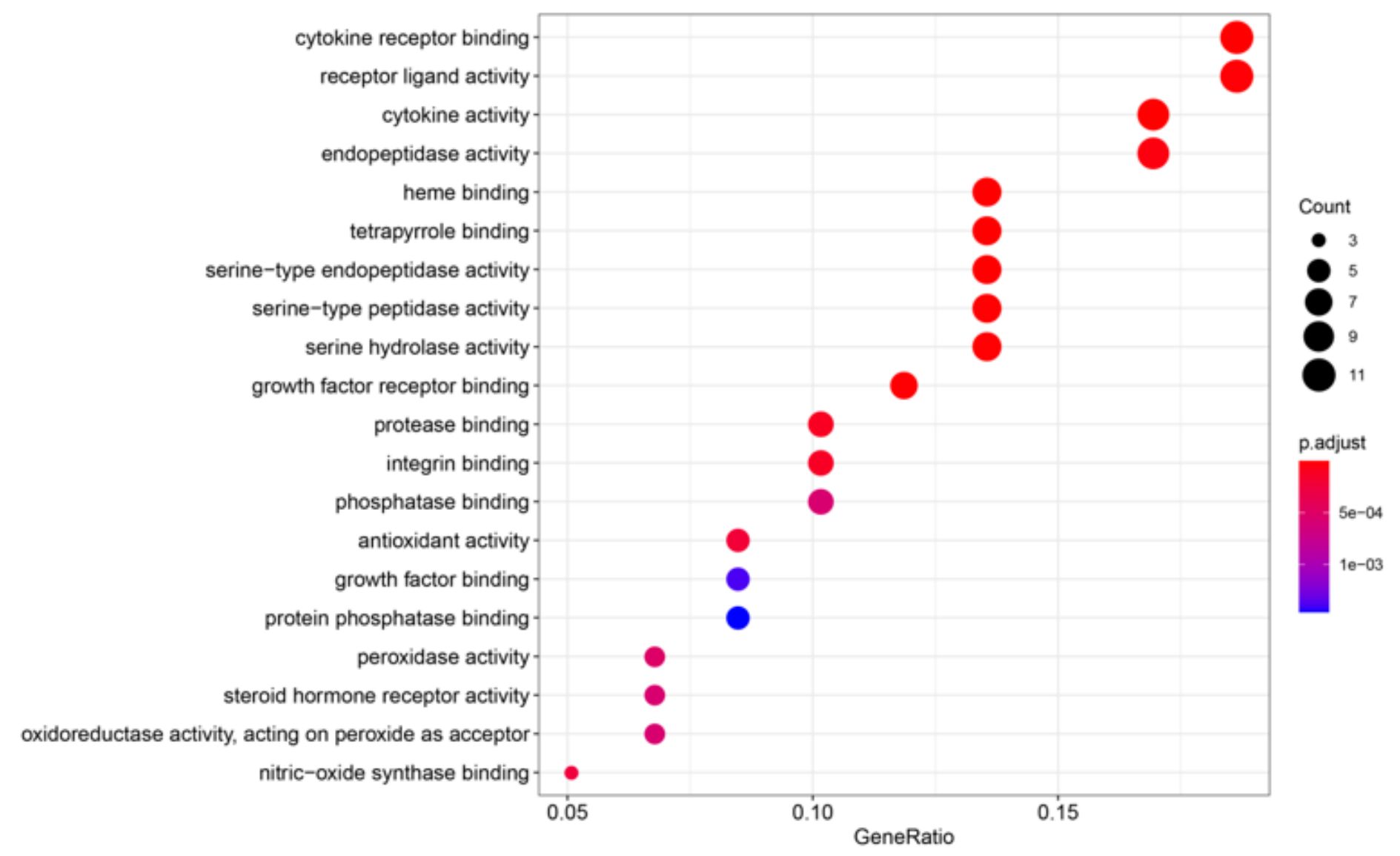

\section{Figure 6}

Representative bubble plots of signalling pathway enrichment analysis of core targets of GQYXD for MF. Gene ratio $=$ count $/$ set size . 
I: Hematoxylin-Eosin

Staining

$(\mathbf{2 0 0 X})$

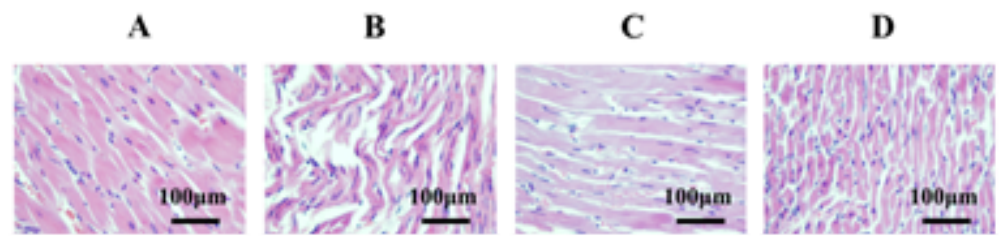

\section{Figure 7}

I) The therapeutic effect of GQYXD on MF assessed by H\&E staining; II) the therapeutic effect of GQYXD in alleviating the deposition of collagen; III-VI) the effect of GQYXD on the expression of AKT1, MAPK8, MAPK14 and PTGS2. A: normal group, B: model group, C: negative control group, D: GQYXD-treated MF group. 


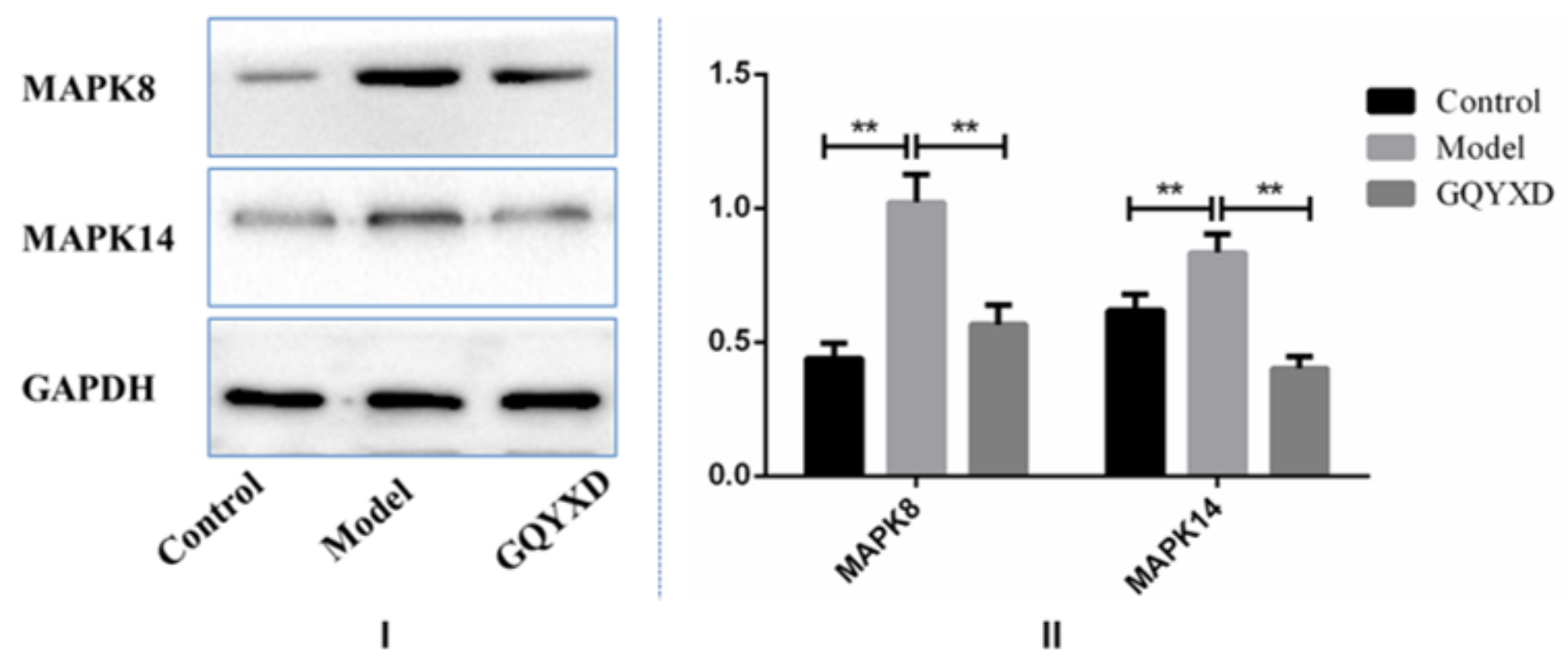

Figure 8

The protein-level expression of MAPK8 and MAPK14 as determined by Western blot; I) protein-level expression of MAPK8 and MAPK14 after isoproterenol induction; II) summary graph of MAPK8 and MAPK14 protein levels in $\mathrm{H} 9 \mathrm{C} 2$ cells.

\section{Figure 9}

The changes in cytoskeleton structure of isoproterenol-induced $\mathrm{H} 9 \mathrm{C} 2$ cells.

\section{Figure 10}

A) The expression of AKT-1 in isoproterenol-induced H9C2 cells. B) The expression of PTGS2 in isoproterenol-induced $\mathrm{H} 9 \mathrm{C} 2$ cells. 